\title{
Scedosporium prolificans
}

National Cancer Institute

\section{Source}

National Cancer Institute. Scedosporium prolificans. NCI Thesaurus. Code C117362.

A species of dematiaceous filamentous fungi in the phylum Ascomycota with small, delicate annellides with a distinct basal swelling peculiar to this species and absent in closely related species. L. prolificans causes opportunistic infection in immunocompetent and immunosuppressed individuals. The species exhibits varying tolerance to currently available antifungal agents. 